\title{
ELECTRON MICROSCOPIC OBSERVATIONS OF POLYSACCHARIDE COMPONENTS IN POLYMYXIN B TREATED OUTER MEMBRANES FROM SERRATIA MARCESCENS*
}

\author{
Douglas A. Weber, Mathew J. Nadakavukaren and Joseph C. Tsang** \\ Departments of Chemistry and Biological Sciences, Illinois State University \\ Normal, Illinois, U. S. A.
}

(Received for publication November 6, 1978)

\begin{abstract}
Treatment of outer membranes of Serratia marcescens with polymyxin B results in the formation of blebs. This effect is thought to be due to the action of the antibiotic on the lipopolysaccharides, proteins, phospholipids or a combination thereof. It is unclear whether this effect is dissociative, degradative or due to an inhibition of the assembly of outer membrane components. Prior studies showed that lipopolysaccharides and polymyxin B form cornplexes, but direct visualization of the in situ action of polymyxin B had not been accomplished. Isolated outer membranes normally exhibit a periodicity of the polysaccharide molecules when stained by the thiosemicarbazide-silver technique. Polymyxin B treated outer membranes display a change in their basic morphology. This effect is very drastic in the sensitive strain as demonstrated by the large gaps in the deposition of the granules in the modified outer membrane structure. Thus it appears that the polysaccharide molecules (probably the lipopolysaccharide) either alone or in association with protein or phospholipids are the primary targets of the antibiotic.
\end{abstract}

Polymyxin B, a cationic cyclicpeptide antibiotic, interacts with various anionic cellular components such as lipopolysaccharides (LPS), ${ }^{1,2)}$ phospholipids (PL), ${ }^{3 \sim 6)}$ deoxyribonucleic acid (DNA), ${ }^{7 \sim 9)}$ and ribosomes $^{8-10)}$ of Gram-negative bacteria. When whole cells are treated with the antibiotic, blebs are formed on the cell surface, ${ }^{7,11 \sim 13)}$ and subsequently, disorganization of the cell envelope occurs if susceptible cells are exposed to a lethal dose of the antibiotic. Although the formation of the blebs has been suggested to be caused by an aggregation and/or complex formation of the antibiotic with outer membrane components such as PL ${ }^{9)}$ and LPS, $\left.{ }^{9}{ }^{14}\right)$ direct in vivo evidence in polymyxin B treated outer membranes has not been available. Recently, the polysaccharide components in the blebs of polymyxin B treated cells of Serratia marcescens have been localized by the silver proteinate method of ThIERY. ${ }^{15)}$ This method involves oxidation by periodic acid (PA) of 1:2 glycol linkages of the polysaccharide to yield aldehydes, which on reaction with thiosemicarbazide (TSC) form hydrazones. Reduction of the silver proteinate reagent by the thiol groups of the hydrazones results in the deposition of silver at the site of original 1:2 glycol linkages. Electron microscopy has been used to observe the deposition of silver as dark granules. In this study we attempted to use this method to observe the effect of polymyxin B on the polysaccharide components in outer membranes isolated from 2 strains of Serratia marcescens.

* Preliminary account of this investigation was presented at the 10th Great Lakes Regional Meeting, American Chemical Society, June 17 19, 1976, Northwestern University, Evanston, Illinois, U.S.A.

** Address all correspondence to Dr. Joseph C. TSANG, Department of Chemistry, Illinois State University, Normal, Illinois, U.S.A. 


\section{Materials and Methods}

Serratia marcescens strain 08 (polymyxin B resistant) and strain Bizio (polymyxin B sensitive) were used in this study. Cells were grown in an enriched medium with aeration at ambient temperature and harvested at an optical density between 0.50 and 0.55 units (after approximately 18 hours of growth). ${ }^{16)}$ Cells from 1 liter of culture medium were treated with $20 \mathrm{mg}$ of polymyxin B sulfate (Burroughs Wellcome) in $75 \mathrm{ml}$ of $0.9 \% \mathrm{NaCl}, \mathrm{pH} 7.8$ for 1 minute at $37^{\circ} \mathrm{C}$. Outer membranes were isolated from treated cells according to the method of OSBORN et al., ${ }^{17)}$ as described by TsANG et al. for cells of $S$. marcescens. ${ }^{18)}$

Ultrathin sections of isolated outer membranes were prepared by using a modified method of ForsBERG et al. ${ }^{19)}$ Samples were embedded in 1\% agar (Difco Noble Special Agar), fixed with $5 \%$ glutaraldehyde and $2 \%$ osmium tetroxide, dehydrated, and embedded in Epon 812. Post section staining was accomplished with uranyl acetate-lead citrate. Staining for polysaccharide components was performed according to the method of THIERY ${ }^{20)}$ as described previously. ${ }^{15}$ ) Thin sections of all samples on gold grids were floated on $1 \%$ PA for 1 hour and washed with distilled water. They were subsequently floated on a $1 \%$ thiosemicarbazide solution in phosphate buffer $(0.05 \mathrm{M}, \mathrm{pH} 7.4)$ and washed with distilled water. Finally the grids were placed on a $1 \%$ aqueous solution of silver proteinate reagent (Electron Microscopy Sciences) for 30 minutes in the dark. All sections were examined in either an Hitachi HU-11A or an RCA EMU-3G electron microscope operating at an accelerating voltage of $50 \mathrm{kV}$.

\section{Results}

The basic morphology of the isolated outer membranes of 2 strains of $S$. marcescens is consistent with the spheroidal shape of the outer membranes of other Gram-negative bacteria. ${ }^{21,22,23)}$ The thin sections of outer membranes stained with uranyl acetate-lead citrate of strain 08 (Fig. 1A) and strain Bizio (Fig. 1B) showed the spheroidal trilaminar structures with smooth periphery. However, the configurations of the morphology of outer membranes isolated from polymyxin B treated cells were modified to some extent. In preparations of strain 08 (Fig. 1C), elongated as well as ribbon-like structures were readily seen. One of the blebs formed on the outer membrane was seen attached to the ribbon-like structure after the outer membrane was disrupted. Fig. 1D shows the high degree of pleiomorphic diversity with numerous shapes in the treated outer membranes of strain Bizio. Although the configurations of most of the structures deviated from the normal spheroidal shape of the untreated outer membranes, the site(s) of the dissociative/degradative action of polymyxin B on the treated outer membrane is not discernible.

When the morphology of untreated outer membranes was observed by the silver proteinate technique of THIERY, ${ }^{20)}$ the normal spheroidal structures were again observed (Figs. 2A and 2B). The silver granules were deposited on the periphery of the double-track structures. The location of the granules, most likely represents the presence of the lipopolysaccharides in the outer membranes. In the treated outer membranes of strain 08 many granules are seen distributed along the periphery of the blebs (Fig. 2C). However, in the strain Bizio the general integrity of the treated outer membranes was lost in addition to a decrease in the number of silver granules deposited on these disrupted membranes. Those granules that were still present were widely dispersed at points along the membrane periphery or on the blebs that were dissociated from the outer membrane proper. Furthermore, the presence of the dark granules in the ribbon-like structures clearly demonstrates the lipopolysaccharide nature of the material released by polymyxin $\mathbf{B}$ treatment. 
Fig. 1. Morphological appearance of outer membranes from Serratia marcescens before and after polymyxin B treatment (stained with uranyl acetate-lead citrate).

The marker bars in all micrographs represent $0.2 \mu \mathrm{m}$.

(A) Untreated outer membrane of the resistant strain 08

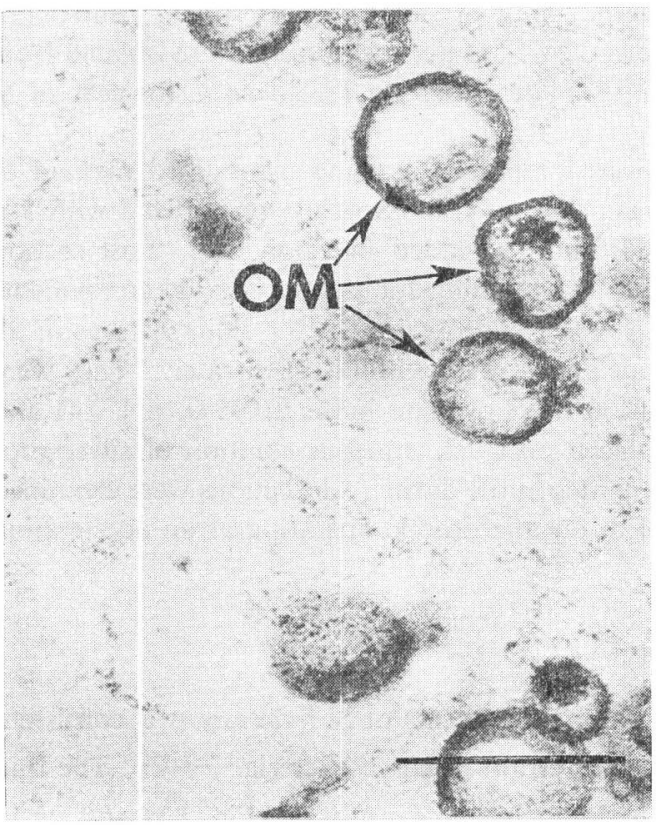

(C) Polymixin B treated resistant strain 08: note the bleb (B) protruding from the elongated outer membrane.

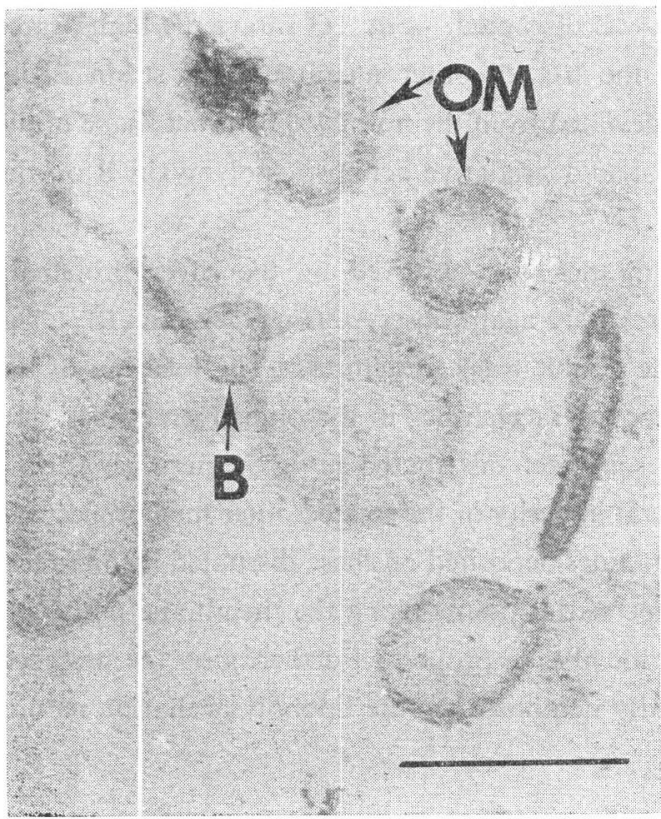

(B) Untreated outer membrane of the sensitive strain Bizio

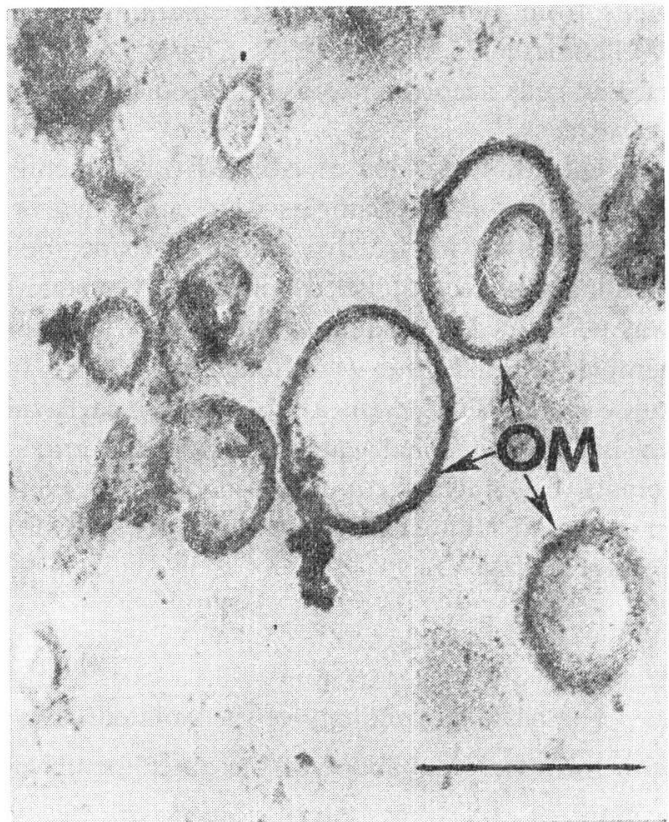

(D) Polymyxin $\mathrm{B}$ treated sensitive strain Bizio: note the bleb (B), the modified outer membrane (M-OM) and the degraded/dissociated (D/D-OM).

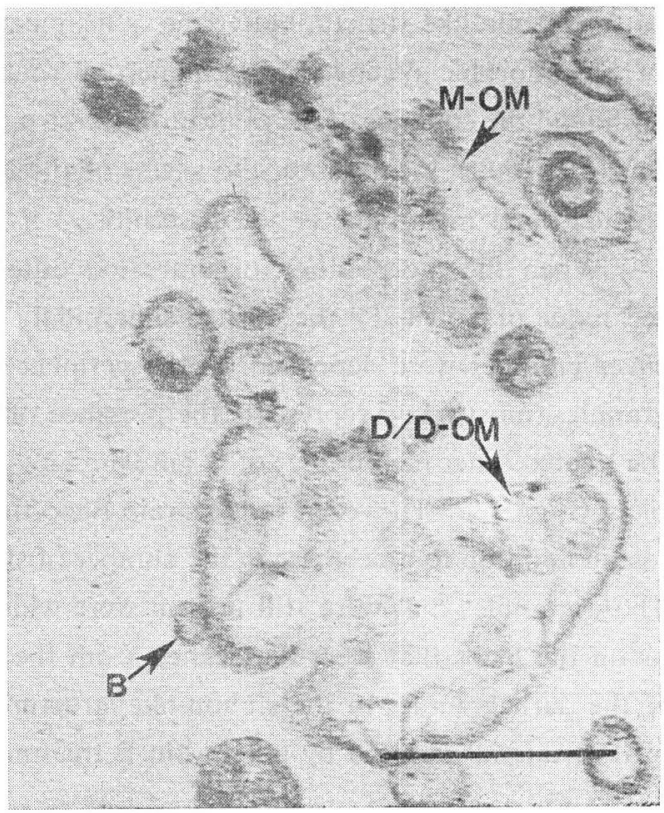


Fig. 2. Morphological appearance of outer membranes from Serratia marcescens before and after polymyxin B treatment (stained by the thiosemicarbazide silver proteinate method).

(A) Untreated outer membranes of the resistant strain 08: note the intense staining along the periphery of the outer membrane (ps).

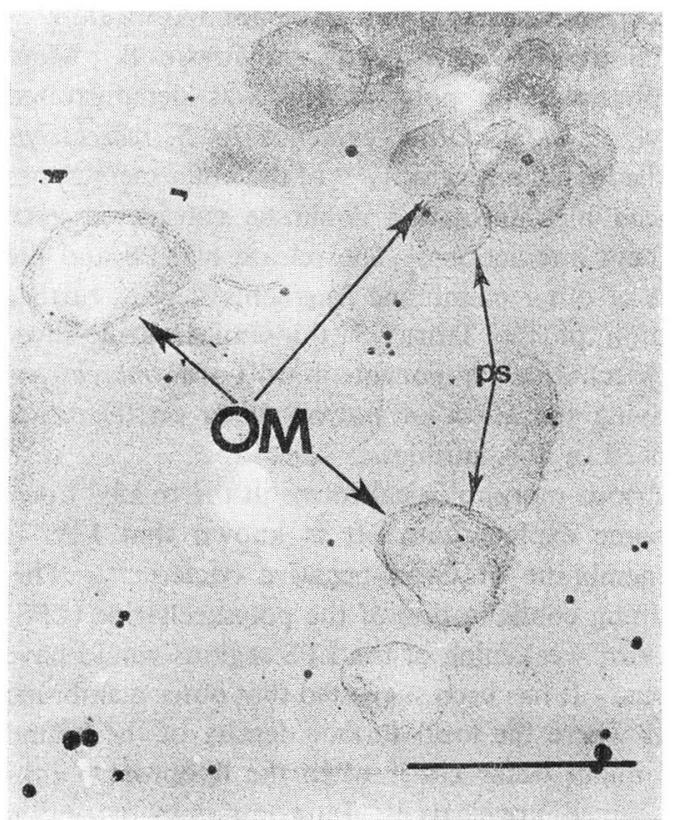

(C) Polymyxin B treated resistant strain 08: note the intense staining of the polysaccharides (ps) that remain after treatment.

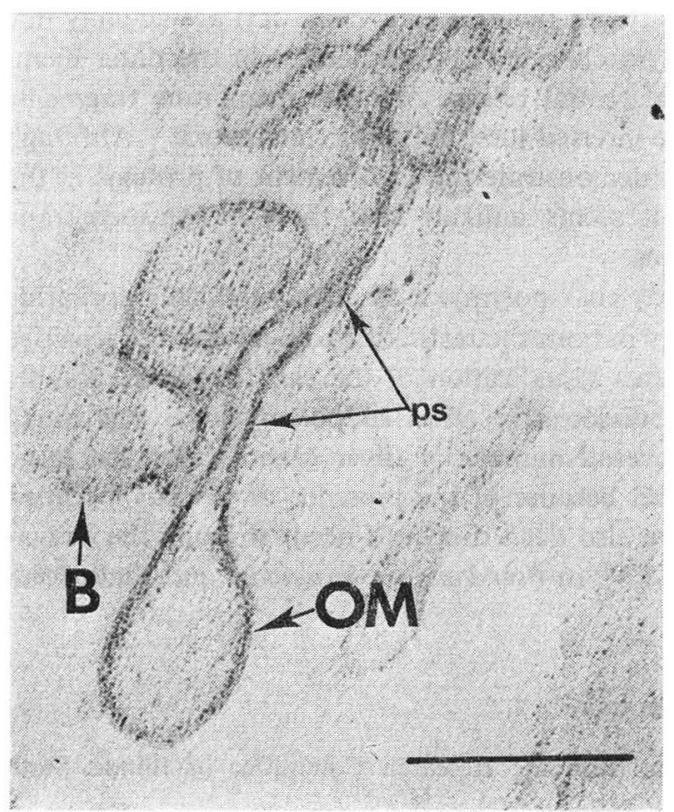

(B) Untreated outer membranes of the sensitive strain Bizio: note the intense staining along the periphery of the outer membrane (ps).

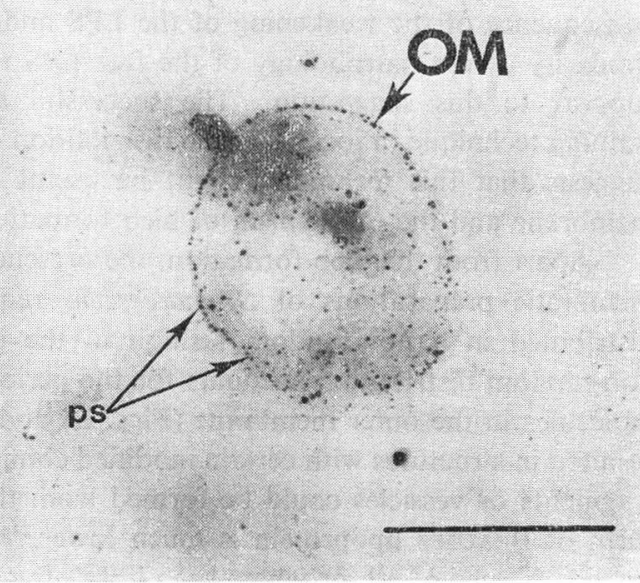

(D) Polymyxin $\mathrm{B}$ treated sensitive strain Bizio: note the lack of stained polysaccharides (no-ps) along the portion of the degraded/dissociated outer membrane (D/D-OM). Also note a few polysaccharide staining sites in a residual membrane fragment (ps).

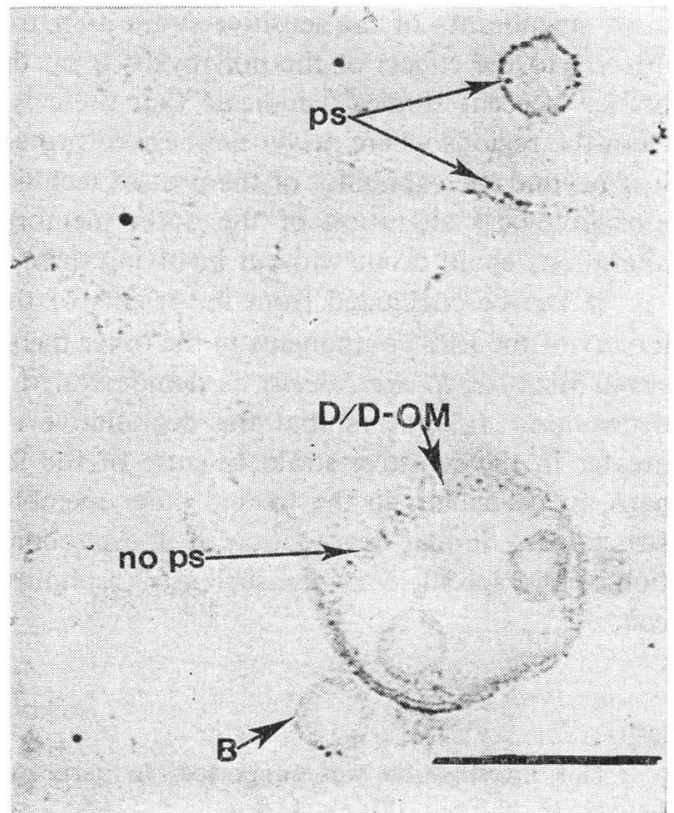




\section{Discussion}

Induction of bleb formation by polymyxin B has been observed in various Gram-negative

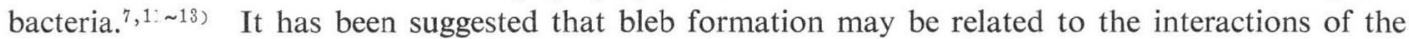
LPS of the outer membranes with polymyxin B. An LPS-like material was first observed to be budding from the outer membrane of a strain of Escherichia coli grown in a lysine-limited medium. ${ }^{24}$ ) LOPES and INNISS ${ }^{25}$ ) described the breakdown of LPS treated in vitro with polymyxin B. More recently, complex formation between the LPS-phospholipid and polymyxin B was demonstrated independently by SCHINDLER and TeUBeR ${ }^{9)}$ and TSANG et al. ${ }^{14)}$ Other evidence in $S$. marcescens included the apparent decrease in yields of LPS $^{16)}$ and the loss of antigenicity ${ }^{26}$ ) of the outer membranes in treated cells. It appears that polymyxin B induced bleb formation could be considered as a consequence of the weakening of the LPS and/or protein interactions. The release of LPS and the similarity of the morphology of the free LPS to those of outer membrane fragments ${ }^{2 i}$ ) gives further support to this suggestion. The successful application of the THIERY ${ }^{20}$ ) thiosemicarbazide-silver staining technique in localizing the distribution of polysaccharide components in cells of $S$. marcescens suggests that this technique might be useful in studying the effect of polymyxin $\mathrm{B}$ on the outer membrane and the mechanism of bleb formation induced by this antibiotic.

Apart from the bleb formation, the presence of various morphological forms in the treated outer membrane preparations of $S$. marcescens requires some explanations. It is known that LPS is distributed in a non-random fashion in the outer membrane of Gram-negative bacteria. ${ }^{29}$ ) This non-random distribution accounts for the periodic staining configuration of the polysaccharide (LPS) molecules in the outer membrane (Figs. 2A and 2B). Any weakening of the LPS regions would have resulted in structures with certain modified configurations. It has been suggested that outer membrane fragments or vessicles could be formed from the areas where the local surface density of the bound form of BRAUN's lipoprotein is much lower. ${ }^{29}$ ) This might occur either when the lipoprotein links are not forrned at all, or where such links after being formed, are cleaved. Interruption by the action of polymyxin B of the insertions of LPS, phospholipids and/or proteins into specific outer membrane zones would have resulted in various sizes and forms of the outer membrane fragments. The presence and intensi:y of the silver deposition at these affected areas provides information not only on the localization of the site of action of polymyxin B with the polysaccharides, but also the extent of the damage of these affected areas. Consistent with this premise is the idea that the large gaps seen in the outer membranes of the sensitive strain after treatment with the antibiotic (Fig. 2D) are actually due directly to the effects of the polymyxin B on the polysaccharide (LPS) molecules in the outer membrane. Recent studies ${ }^{30)}$ indicate that there is a preferential release of outer membrane fragments from the regions where newly synthesized proteins are inserted into the outer membrane. Although it is beyond the capability of the present technique to demonstrate the involvement of proteins in the morphological alteration of the outer membranes, it seems unlikely that these major membrane alterations could occur without involving these proteins.

It can be concluded from the results of this study that polymyxin B affects the polysaccharide moiety of the LPS components in the outer membranes in both the resistant strain 08 and the sensitive strain Bizio of $S$. marcescens as demonstrated by direct visualization of the electron dense granule deposition. It appears that the degradative and/or dissociative effect of polymyxin $\mathbf{B}$ was much greater in the sensitive strain because of the fewer overall number of silver granules and the large gaps, in particular, in the treated outer membrane and because of the presence of various modified structures. Similar degradation of phospholipids have also been shown to occur through the activation of OM specific enzymes, such as phospholipase $\mathrm{A}_{1}{ }^{31,32)}$ in Pseudomonas aeruginosa and Escherichia coli.

\section{Acknowledgements}

This investigation was supported, in part, by grants from the Research Committee of Illinois State University, Normal, Illinois, U.S.A. 


\section{References}

1) Bader, J. \& M. Teuber: Action of polymyxin B on bacterial membranes. I. Binding to the Oantigenic lipopolysaccharide on Salmonella typhimurium. Z. Naturforsch. 28c: 422 430, 1973

2) RifKIND, D.: Studies on the interaction between endotoxin and polymyxins. J. Infect. Dis. 177: 433 438, 1967

3) Hsuchen, C. C. \& D. S. Ferngold: Locus of divalent cation inhibition of the bacterial action of polymyxin B. Antimicr. Agents \& Chemoth. 2: 331 338, 1972

4) Imai, M.; K. Inoue \& S. NoJima: Effect of polymyxin B on liposomal membranes derived from Escherichia coli lipids. Biochim. Biophys. Acta 375: 130 137, 1975

5) Teuber, M. \& J. BAder: Interaction of polymyxin with outer membrane of Salmonella typhimurium binding to isolated lipopolysaccharides and lipid A. Abst. Com. Meeting Feb. Europ. Biochem. Soc. 8: 26, 1972

6) Teuber, M.: Action of polymyxin B on bacterial membranes. II. Formation of lipophilic complexes with phosphatidic acid and phosphatidylglycerol. Z. Naturforsch. 26: 476 477, 1973

7) Handley, P. S.; L. B. Quesnel \& M. Sturgis: Ultrastructural changes produced in Proteus vulgaris by synergistic combination of colistin and sulphdizine. Microbios 10: 211 233, 1974

8) Nakajima, K. \& J. Kawamata: Studies on the mechanisms of action of colistin. I. Formation of an insoluble complex with nucleic acids. Biken J. 8: 225 231, 1965

9) Schindler, P. R. G. \& N. Teuber: Action of polymyxin B on bacterial membranes: Morphological changes in the cytoplasm and in the outer membrane of Salmonella typhimurium and Escherichia coli. Antimicr. Agents \& Chemoth. 8: 95 104, 1975

10) Nakajima, K. \& J. Kawamata: Studies on the mechanisms of action of colistin. II. Precipitation of Escherichia coli ribosome with colistin. Biken J. 9: 45 50, 1966

11) Suganuma, S.; K. Hara, T. Kishida, K. Nakajima \& J. Kawamata: Cytological changes of Escherichia coli caused by polymyxin B. Biken J. 11: 149 155, 1968

12) WAhn, K.; G. Lutsch, T. RoKstroh \& K. SAPF: Morphological and physiological investigations on the action of polymyxin B on Escherichia coli. Arch. Mikrobiol. 63: 103 116, 1968

13) Korke, M.; K. Iida \& T. Matsuo: Electron microscopic studies on some Proteus strains. Acta Path. Microbiol. Scand. 80: 641 649, 1969

14) Tsang, J. C.; D. A. Weber \& D. A. Brown: Evidences for complex formation between polymyxin B and lipopolysaccharides from Serratia marcescens. J. Antibiotics 29: 735 742, 1976

15) Weber, C. A.; M. J. Nadakavukaren \& J. C. Tsang: Localization of polysaccharide components in polymyxin B treated cells of Serratia marcescens. J. Antibiotics 31: 732 735, 1978

16) Tsang, J. C.; D. A. Brown \& D. A. Weber: Effects of polymyxin B on cell morphology and lipopolysaccharide composition of Serratia marcescens. Microbios 14: 43 54, 1975

17) Osborn, J.; J. Gander, E. Parisi \& J. Carson: Mechanism of assembly of the outer membrane of Salmonella typhi: Isolation and characterization of inner membrane and outer membrane. J. Biol. Chem. 247: 3962 3972, 1972

18) Tsang, J. C.; D. A. Brown \& D. A. Kranz: Detection of lipopolysaccharide components in isolated outer membranes of Serratia marcescens. Microbios Letters 1: 209 217, 1976

19) Forsberg, C. W.; J. W. Costerton \& R. A. Macleod: Separation and localization of cell wall layers of a gram-negative bacterium. J. Bacteriol. 104: 1338 1353, 1970

20) Thiery, J. P.: Mise en evidence des polysaccharides sur corpes fines en microscopie electronique. J. Microscopie 6: $987 \sim 1018,1967$

21) Hasin, M.; S. Rottem \& S. Razin: The outer membrane of Proteus mirabilis. I. Isolation and characterization of the outer and cytoplasmic membrane fractions. Biochim. Biophys. Acta 375: 381 394, 1975

22) Rottem, S.; M. Hasin \& S. RazIn: The outer membrane of Proteus mirabilis. II. The extractable lipid fraction and electron paramagnetic resonance analysis of the outer and cytoplasmic membranes. Biochim. Biophys. Acta 375: 395 405, 1975

23) Thorne, K; J. I. Kareen, M. J. Thornley \& A. M. Glauert: Chemical analysis of the outer membrane and other layers of the cell envelope Acinetobacter sp. J. Bacteriol. 116: 410 417, 1973

24) Work, E.; K. W. KNOx \& M. VeSK: The chemistry and electron microscopy of an extracellular lipopolysaccharide from Escherichia coli. Ann. N.Y. Acad. Sci. 133: 438 449, 1966

25) Lopes, J. \& W. E. InNISS: Electron microscopy of effects of polymyxin on Escherichia coli lipopolysaccharide. J. Bacteriol. 100: 1128 1130, 1969 
26) Brown, D. A. \& J. C. Tsang: Effect of polymyxin B on the antigenicity of outer membrane from Serratia marcescens. Microbios Letters 2: 189 196, 1976

27) Weber, D. A.; M. J. Nadakavukaren \& J. C. Tsang: The effect of polymyxin B on outer membranes of Serratia marcescens: Morphological alterations of the outer membranes and their lipopolysaccharide components. Microbios 17: 149 161, 1976

28) LeIVE, L.: Domains involving non-random distribution of lipopolysaccharide in the outer membrane of Escherichia coli. Proc. Natl. Acad. Sci. (U.S.) 74: 5065 5068, 1977

29) Hoeks ra, D.; J. W. van Der LaAn, L. De LeiJ \& B. Witholt: Release of outer membrane fragments from normally growing Escherichia coli. Biochim. Biophys. Acta 455: 889 899, 1976

30) Mug-Opstelten, D. \& B. Witholt: Preferential release of new outer membrane fragments by exponentially growing Escherichia coli. Biochim. Biophys. Acta 508: 287 295, 1978

31) Kusanio, T.; K. Izaki \& H. TAKahashr: Degradation of phospholipid in Pseudomonas aeruginosa induced by polymyxin B. J. Antibiotics 28: 689 695, 1975

32) Kusanio, T.; K. Izaki \& H. TAKahashi: In vivo activation by polymyxin $\mathrm{B}$ of phospholipase from Pseudomonas aeruginosa and Escherichia coli. J. Antibiotics 29: 674 675, 1976 